\title{
Proximity-induced topological state in graphene
}

\author{
Igor Popov, Mauro Mantega, Awadhesh Narayan, ${ }^{*}$ and Stefano Sanvito \\ School of Physics, AMBER and CRANN, Trinity College, Dublin 2, Ireland \\ (Received 19 August 2013; revised manuscript received 30 June 2014; published 15 July 2014)
}

\begin{abstract}
The appearance of topologically protected states at the surface of an ordinary insulator is a rare occurrence and to date only a handful of materials are known for having this property. An intriguing question concerns the possibility of forming topologically protected interfaces between different materials. Here we propose that a topological phase can be transferred to graphene by proximity with the three-dimensional topological insulator $\mathrm{Bi}_{2} \mathrm{Se}_{3}$. By using density functional and transport theory, we prove that, at the verge of the chemical bond formation, a hybrid state forms at the graphene $/ \mathrm{Bi}_{2} \mathrm{Se}_{3}$ interface. The state has Dirac-cone-like dispersion at the $\Gamma$ point and a well defined helical spin texture, indicating its topologically protected nature. This demonstrates that proximity can transfer the topological phase from $\mathrm{Bi}_{2} \mathrm{Se}_{3}$ to graphene.
\end{abstract}

DOI: 10.1103/PhysRevB.90.035418

PACS number(s): 72.80.Vp, 61.46.Km, 62.25.-g

\section{INTRODUCTION}

Topological insulators (TIs) are a recently discovered class of materials presenting an electronic band gap in the bulk and metallic edge states at their surfaces [1-3]. The peculiarity is that the edge states are protected against electron scattering to impurities, so that they can act as perfectly ballistic conductors $[1,4]$. To date only a handful of TIs have been synthesized and high-throughput materials screening has indicated that only few more may be fabricated by straining existing inorganic compounds [5]. Intriguingly, with the only exception of $\mathrm{CdTe} / \mathrm{HgTe}$ quantum wells [6], there are no reports of two-dimensional (2D) TIs. Thus, at the moment, we have at hand only three-dimensional (3D) TIs with 2D edge states. Yet, one may wonder whether we have exhausted all the possibilities for creating useful topologically protected states in materials.

A particularly intriguing prospect is that of using the interaction between different materials to create hybrid interfaces with topological properties. For instance, depositing normal semiconductors on top of 3D topological insulators may result in a structure that under certain conditions exhibits topologically protected interface states [7]. An even more attractive prospect is that of using that protocol for transferring topologically protected states to graphene $[8,9]$. Since graphene-based transistors have been already demonstrated [10], one could then speculate on having graphene logic elements connected by topological-graphene interconnects, i.e., on realizing an all graphene high-performance logic circuitry. A major advantage of such strategy is its full compatiblity with $2 \mathrm{D}$ patterning.

Several proposals have been already brought forward for making graphene topological [11]. Indeed, one of the first TI models was based on a staggered hexagonal lattice with a helicity-dependent complex hopping parameter, simulating spin-orbit interaction [12]. However, since the spin-orbit coupling in graphene is tiny, a topological phase may be induced only by strongly perturbing the graphene electrostatic potential, for instance, by adsorbing heavy ions on top of the sheet [13]. Importantly, although theoretically sound, such

\footnotetext{
*narayaa@tcd.ie
}

a proposal requires ultra-accurate fabrication precision and appears rather challenging in practice. Here, we suggest a completely different approach: we introduce topologically protected states in graphene by proximity with a latticecommensurate 3D TI. This happens at the graphene/ $\mathrm{Bi}_{2} \mathrm{Se}_{3}$ interface, a composite which was synthesized about two years ago [14-16], but whose electronic structure still remains unclear.

\section{COMPUTATIONAL METHODS}

Calculations are performed by density functional theory (DFT) as implemented in the VASP code $[17,18]$. We use the Perdew-Burke-Ernzerhof form of the generalized gradient approximation [19] and the core electrons are described by projector-augmented-wave pseudopotentials [20]. The $k$-space integration spans a $11 \times 11 \times 1$ Monkhorst-Pack mesh in the irreducible Brillouin zone and the plane-waves cutoff is $400 \mathrm{eV}$.

The geometry of the structure investigated is shown in Fig. 1. We consider a $\mathrm{Bi}_{2} \mathrm{Se}_{3}$ slab containing three quintuple layers (QLs), for which the tensile stress is minimal among the experimentally investigated $\mathrm{Bi}_{2} \mathrm{Se}_{3} /$ graphene composites [14]. The $\mathrm{Bi}_{2} \mathrm{Se}_{3}$ unit cell is commensurate with three graphene unit cells, hence the elementary unit cell of the composite contains an entire carbon ring. The contacting Se atom is placed at the graphene hollow site (in the center of the ring). The in-plane lattice parameter is $4.26 \AA$, which is only $2.3 \%$ larger than the lattice parameter of bulk $\mathrm{Bi}_{2} \mathrm{Se}_{3}$ [21]; the one perpendicular to the interface is instead $40 \AA$ (there is a vacuum region $>10 \AA$ between cells periodic replica). We have also investigated a second geometry where the carbon atoms are on top of Se. This, however, is not energetically favorable and it has not been considered in the rest of the paper. Interestingly, both interface structures present rather similar trends in the electronic structure properties.

\section{RESULTS AND DISCUSSION}

Let us begin by investigating the evolution of the graphene band gap with the graphene $/ \mathrm{Bi}_{2} \mathrm{Se}_{3}$ distance, $d$. We assign an electronic band to a given material by projecting the energy and $k$-dependent wave function onto spherical harmonics 


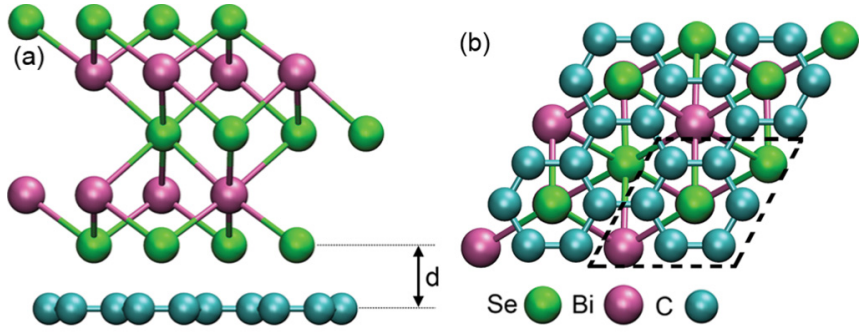

(c)

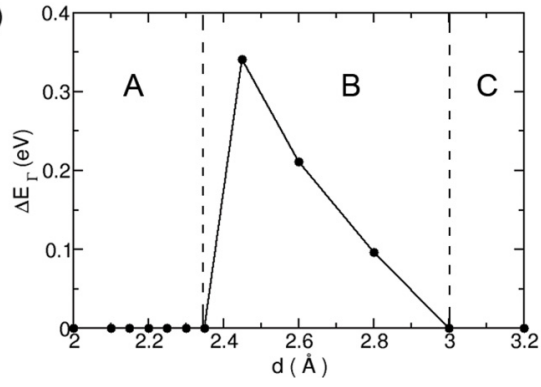

FIG. 1. (Color online) Side (a) and top view (b) of the graphene/ $\mathrm{Bi}_{2} \mathrm{Se}_{3}$ interface. The graphene- $\mathrm{Bi}_{2} \mathrm{Se}_{3}$ separation is $d$. In (c), we report the graphene electronic band gap (see text) as a function of $d$.

centered around particular atoms [22]. We define the graphene electronic band gap from those bands located near the charge neutral point of free-standing graphene and having dominant $\mathrm{C}$ character. In Fig. 1(c), one can identify three different regions. For $d>3 \AA$ [region C], graphene has no band gap. This is expected since for large separations the interaction is weak and the band structure of the composite is the superposition of those of the constituents. As such graphene remains a zero-gap semiconductor. Region B is characterized by the opening of the graphene band-gap. The gap increases monotonically from $d=3 \AA$ and it reaches a maximum $(0.34 \mathrm{eV})$ for $d=2.45 \AA$. A further reduction in $d$ (region $\mathrm{A}$ ) closes the gap, which remains close up to $d=2 \AA$.

Next, we analyze in Fig. 2 the nature of the graphene bands around the Fermi level, $E_{\mathrm{F}}$, as a function of $d$. For $d>3 \AA$ [Fig. 2(a)], the composite features two superimposed band structures corresponding to those of graphene and $\mathrm{Bi}_{2} \mathrm{Se}_{3}$, respectively. At such large separation, there is no wave function overlap between graphene and $\mathrm{Bi}_{2} \mathrm{Se}_{3}$, leaving the two materials electronically decoupled. The graphene's linearly dispersive bands (in blue in Fig. 2) at each of the valleys (Dirac K-points) are twofold spin-degenerate, with the $\pi\left(E<E_{\mathrm{F}}\right)$ and $\pi^{*}\left(E>E_{\mathrm{F}}\right)$ bands just touching each other. The $\mathrm{Bi}_{2} \mathrm{Se}_{3}$ surface states (green bands in Fig. 2) cross $E_{\mathrm{F}}$ at the $\Gamma$ point.

Decreasing $d$ below $3 \AA$ causes a band-gap opening between the $\pi$ and $\pi^{*}$ bands [see Fig. 2(b), $d=2.6 \AA$ ]. Now the graphene valleys are placed together with the $\mathrm{Bi}_{2} \mathrm{Se}_{3}$ surface states around the $\Gamma$ point due to the bands folding in the supercell structure. As the graphene electronic gap increases further upon a reduction of $d$ [see $d=2.4 \AA$, Fig. 2(c)], the $\pi^{*}$ cone lifts up in energy but the tip of the $\pi$ one remains pinned at $E_{\mathrm{F}}$. The topologically protected surface states of $\mathrm{Bi}_{2} \mathrm{Se}_{3}$ (one per surface) are positioned in the vicinity of the tip of the $\pi$ cone. For separations $d>2.6 \AA$, these surface states form a doubly degenerate state since the two surfaces are equivalent for the unperturbed TI slab. For distances $d \leqslant 2.6 \AA$, the symmetry of the TI slab breaks due to the vicinity of the graphene layer and the degeneracy of the surface states is
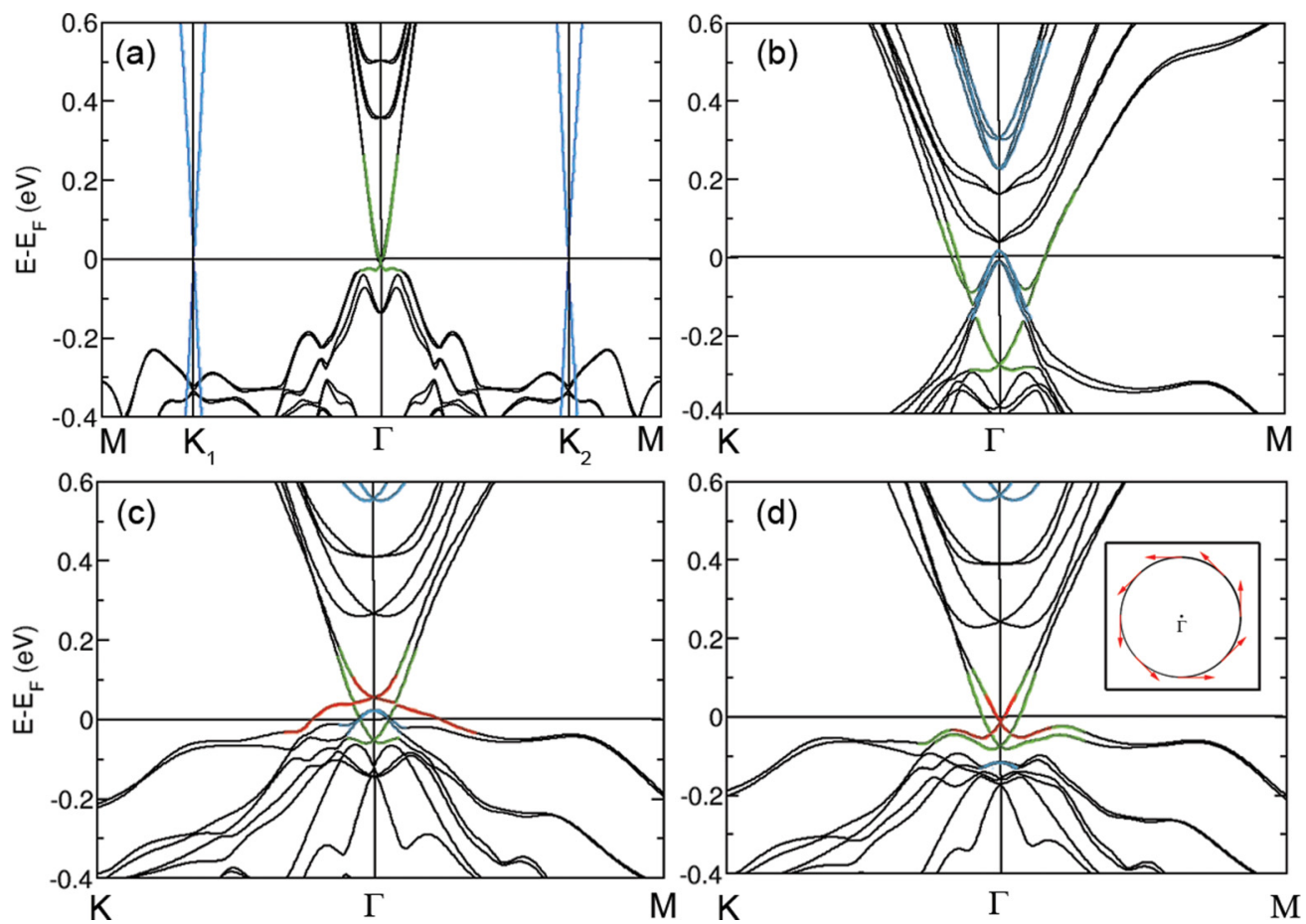

FIG. 2. (Color online) Evolution of band structure of the graphene/ $\mathrm{Bi}_{2} \mathrm{Se}_{3}$ composite as a function of the separation $d$ between the two constituents. In (a), (b), (c), and (d), we present the band-structure for $d=3.0,2.6,2.3$, and $2.2 \AA$, respectively. Black and green bands are bulk and surface states of $\mathrm{Bi}_{2} \mathrm{Se}_{3}$, blue bands are graphene bands, while the red ones represent hybrid states. The inset in (d) illustrates the spin-texture of the mixed state at $0.05 \mathrm{eV}$ above $E_{\mathrm{F}}$. Note the different $k$-point samplings for $d=3.0 \AA$. 
lifted. The TI surface state in contact with graphene moves up in energy at $\Gamma$ by about $0.11 \mathrm{eV}$ for $d=2.3 \AA$. Importantly, the graphene states that are pinned at $E_{\mathrm{F}}$ start to couple with the surface state and, for $d \leqslant 2.3 \AA$, the fourfold degeneracy of the $\pi$ cone is lifted. Here, the pure graphene bands forming the $\pi$ cone are pushed down in energy and only the mixed graphene/ $\mathrm{Bi}_{2} \mathrm{Se}_{3}$ band [red in Fig. 2(d)] and the $\mathrm{Bi}_{2} \mathrm{Se}_{3}$ surface state at the opposite side of the interface (placed directly beneath the mixed band at $\Gamma$ ) cross $E_{\mathrm{F}}$.

Intriguingly, such newly formed mixed graphene/ $\mathrm{Bi}_{2} \mathrm{Se}_{3}$ band presents a helical spin-texture, demonstrated in the inset of Fig. 2(d). This is a sufficient condition for disabling back-scattering of charge carriers [1], and it is not the case in a pristine graphene sheet. In fact, defects in graphene allow hopping of charge carriers between two valleys, which causes back-scattering due to their opposite winding numbers. In contrast, hopping is impossible in systems with only one valley and a helical spin-texture, which is the case for graphene $/ \mathrm{Bi}_{2} \mathrm{Se}_{3}$. Note, however, that the existence of the helical spin texture and the lack of back-scattering do not necessarily mean topological protection of the material's electronic state [23].

The supercell structure causes the folding of the second Brillouin zone (BZ) of primitive graphene into the first and consequently the migration of the graphene valleys from $\mathrm{K}$ to $\Gamma$. Thus two fourfold degenerate cones touching at $E_{\mathrm{F}}$ are formed. The bands from the two valleys have opposite topological charges, which causes their mutual annihilation [24]. This manifests itself in the opening in graphene of a band gap [see Figs. 2(b) and 2(c)], much larger than that estimated for thallium adatom deposition [13].

After having determined the emergence of a mixed graphene $/ \mathrm{Bi}_{2} \mathrm{Se}_{3}$ band, we now analyze in detail its electronic properties. The electron density integrated over a narrow energy region around $E_{\mathrm{F}}$ and projected over the mixed state is shown in Fig. 3(b). This is clearly localized over graphene and, to a smaller degree, over the Se atoms in contact to graphene. Since such state presents dominating C- $p_{z}$ and Se- $p_{z}$ orbital contributions and it is delocalized in the plane of the interface, it presents a $\pi$ conjugation. A more quantitative insight is obtained by plotting the charge density averaged over planes parallel to the interface [Fig. 3(c)]. This shows that, while the contribution to the electron density originating from the bulk is small, a much larger portion is provided by the two surface states at both sides of the composite. At the free $\mathrm{Bi}_{2} \mathrm{Se}_{3}$ surface, the surface state is distributed mainly over the first four atomic layers [Fig. 3(a)]. In contrast, at the graphene/ $/ \mathrm{Bi}_{2} \mathrm{Se}_{3}$ interface, the electron density migrates from the TI to graphene. Notably, this feature resembles closely the one reported for the interface between the normal metal $\mathrm{Sb}_{2} \mathrm{Se}_{3}$ and the $\mathrm{TI} \mathrm{Bi}_{2} \mathrm{Se}_{3}$ [25]. Also, the behavior is similar to the topologization of $\mathrm{Zn} M(M=\mathrm{S}$, $\mathrm{Se}, \mathrm{Te}$ ) upon deposition on $\mathrm{Bi}_{2} \mathrm{Se}_{3}$ [7].

Let us now spend a few words on the possibility of inducing a topological state in graphene due to its proximity and bonding to $\mathrm{Bi}_{2} \mathrm{Se}_{3}$. Firstly, we wish to point out that our results do not indicate that graphene converts into a $2 \mathrm{D}$ topological insulator upon its deposition on $\mathrm{Bi}_{2} \mathrm{Se}_{3}$, but simply that a topologically protected hybrid state is formed. A 2D TI is an insulator in the bulk presenting topologically protected $1 \mathrm{D}$ states at the edges of a ribbon, as in the case of $\mathrm{Bi}$ thin films on $\mathrm{Bi}_{2} \mathrm{Te}_{3}$

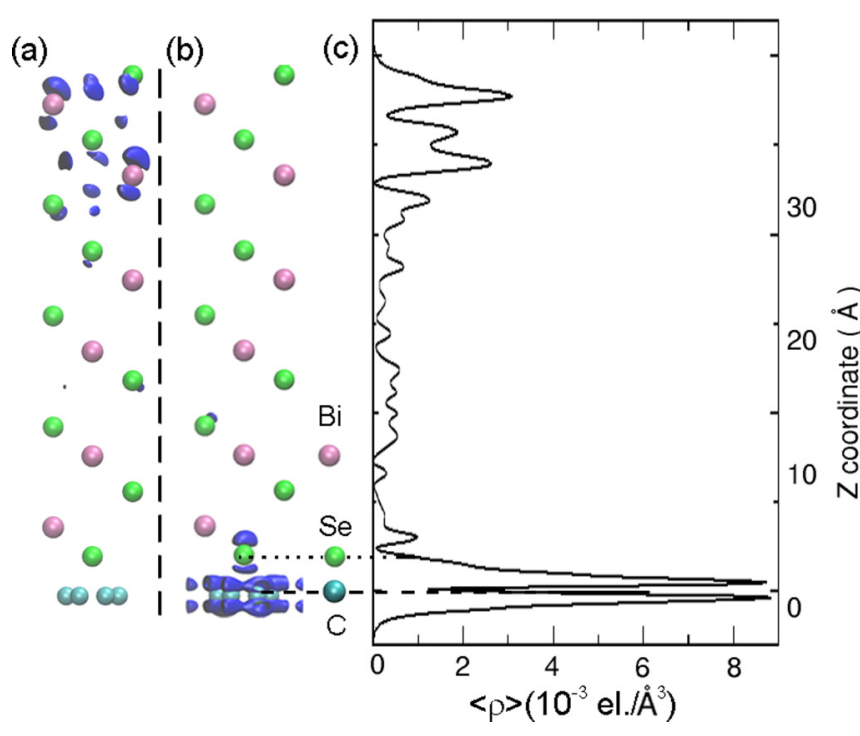

FIG. 3. (Color online) Charge density associated to the $\mathrm{Bi}_{2} \mathrm{Se}_{3}$ surface opposite to the graphene/ $\mathrm{Bi}_{2} \mathrm{Se}_{3}$ interface (a) and the mixed interface state (b) obtained for $d=2.2 \AA$ at $\Gamma$. (c) Sum of the two charge densities averaged over a plane parallel to the interface.

surface [26,27]. In contrast, here the Fermi surface of graphene undergoes a transition from a zero-band-gap semiconducting phase (region C), prone to gap opening due to defects and impurities, to a topologically protected metallic phase (region A) via an insulating phase (region B). A fundamental property of 3D TIs is the existence of an odd number of surface bands around $E_{\mathrm{F}}$. Another property is that the topology of the surface states is such to connect the bulk valence band to the conduction one due to the parity inversion originated by the strong spin-orbit coupling. In the graphene/ $\mathrm{Bi}_{2} \mathrm{Se}_{3}$ complex, only one conical band is present at the Fermi level. Importantly, this band belongs to the surface state of $\mathrm{Bi}_{2} \mathrm{Se}_{3}$ with considerable contribution of graphene around $E_{\mathrm{F}}$, while the other parts of the state (in particular the ends that connect to the valance and the conduction bulk TI states) still fully belong to the TI. Thus the topologically protected surface TI state can be understood as a carrier of the induced graphene states, and the intrinsic topological protection of TI surface state provides the robustness to graphene as well.

The topologically protected hybrid state does not simply correspond to the penetration of the one of the $\mathrm{Bi}_{2} \mathrm{Se}_{3}$ edge states into graphene. In that situation, interaction between $\mathrm{Bi}_{2} \mathrm{Se}_{3}$ and graphene is not present. In contrast, here, the interaction is strong and, in fact, as $d$ decreases first, it is responsible for the opening of a band gap in graphene and then for creating the topologically protected hybrid state. This behavior is very similar to that of the $\mathrm{Sb}_{2} \mathrm{Se}_{3} / \mathrm{Bi}_{2} \mathrm{Se}_{3}$ [25] and the $\mathrm{Zn} M / \mathrm{Bi}_{2} \mathrm{Se}_{3}$ ( $M=\mathrm{S}, \mathrm{Se}, \mathrm{Te})$ [7] interfaces, in which a topological state is transferred to the normal metal because of proximity.

As a final characterization of the hybrid state, we have probed its scattering properties. In particular, we have performed transport calculations, with SMEAGOL code [28], for the composite along the direction parallel to the graphene sheet. Note that SMEAGOL provides an electronic structure for the composite essentially identical to that obtained with VASP. We have then calculated the system conductance for a defect-free 
(a)

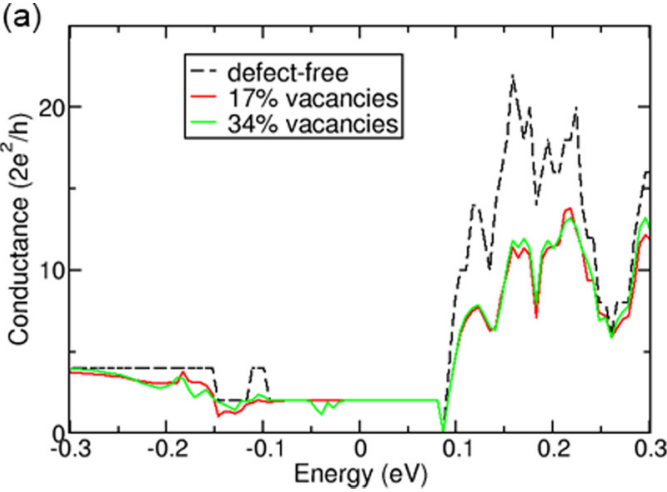

(b)

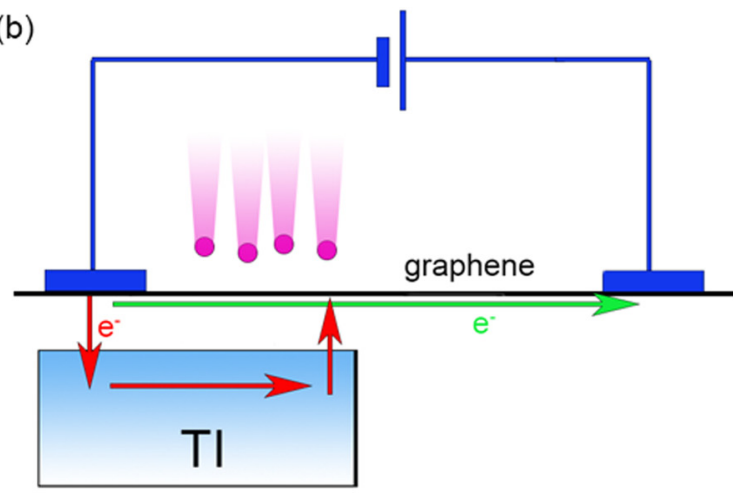

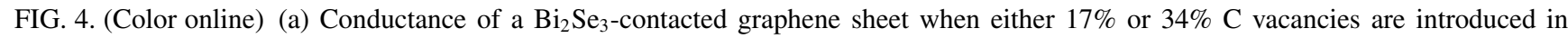
graphene as compared to the conductance of a defect-free layer. (b) Schematic overview of a proposed experimental setup, which may prove the transfer of a topologically protected state from TI to graphene.

system and for the case where approximately $17 \%$ or $33 \%$ vacancies counting atoms are introduced along the direction perpendicular to the transport [see Fig. 4(a)]. Note that these are extremely large concentrations and here they serve the purpose to prove the topological protection of the surface state. From Fig. 4(a), one can observe that $17 \%$ of vacancies do not affect the conductance around $E_{\mathrm{F}}$ indicating that the state is indeed strongly protected against back-scattering. Even for a $33 \%$ concentration, little reduction of the conductance is found at around $E_{\mathrm{F}}$, although the graphene layer is almost cut in two parts. Note, however, that the inclusion of impurities reduces significantly the conductance for energies away from the Fermi level, i.e., away the topologically protected part of the graphene spectrum. This demonstrates that the transport is indeed through graphene and it is protected against back-scattering at around the Fermi level.

Concluding, we would like to propose an experiment, which may prove the transfer of the topologically protected state to graphene. A schematic view of the proposed setup is shown in Fig. 4(b), in which a graphene sheet is contacted only in part to $\mathrm{Bi}_{2} \mathrm{Se}_{3}$, while the rest remains free-standing. Defects are then introduced in graphene only at the contacting region for instance by electron or ion irradiation [29]. If there exists topological protection in graphene at the contact region, the electronic transport through such system will not show a conductance reduction relative to that of the defect-free case. One may still argue that the transport through the irradiated region is via the topologically protected surface state of $\mathrm{Bi}_{2} \mathrm{Se}_{3}$ rather than through the defective graphene. However, in this case, the charge carriers need to hop between the TI and the contacting graphene [red arrows in Fig. 4(b)] in order to continue their flow through the bare graphene and close the electric circuit. This will degrade the conductance. In contrast, if the transport is carried solely by states of the (defected) graphene, the effects of hopping will be eliminated by the proposed geometry setup.

Throughout the paper, we have presented results as a function of the graphene/ $\mathrm{Bi}_{2} \mathrm{Se}_{3}$ distance, therefore we would like to close this section by briefly discussing what equilibrium distance one can expect. Unfortunately, this turns out to be a difficult problem. The exact binding distance is determined by a balance between covalent and van der Waals forces.
These latter ones are not captured by DFT local/semilocal exchange and correlation functionals and, in fact, we find that the two materials do not bind when the calculation is done at the generalized gradient approximation level [19]. This contrasts reality where the graphene $/ \mathrm{Bi}_{2} \mathrm{Se}_{3}$ exists as it has been experimentally fabricated by few groups [14-16]. Unfortunately, the inclusion of van der Waals forces at the level of local DFT [30] does not improve the situation, as screening prevents an accurate evaluation of the binding energy in layered compounds [31]. However, we expect the equilibrium graphene/ $\mathrm{Bi}_{2} \mathrm{Se}_{3}$ distance to be close to the sum of Se and C covalent radii, which amounts to $2 \AA$. This is well within region A [see Fig. 1(c)], i.e., when the hybrid surface state forms. Besides, external pressure may be introduced for tuning the desired separation.

\section{CONCLUSIONS}

In conclusion, we have investigated the electronic properties of graphene in contact to $\mathrm{Bi}_{2} \mathrm{Se}_{3}$. Three phases have been identified, depending on the graphene/ $\mathrm{Bi}_{2} \mathrm{Se}_{3}$ separation. For $d>3.0 \AA$, the electronic structure of the composite is simply the superposition of those of the constituents. The second phase, obtained for $2.4 \AA \leqslant d \leqslant 3.0 \AA$, witnesses the opening of a band gap in graphene, due to the annihilation of graphene states with opposite winding numbers. The third phase, when graphene and $\mathrm{Bi}_{2} \mathrm{Se}_{3}$ chemically bind, is the most interesting, as a topologically protected state with charge distribution mostly localized on graphene forms.

Recently, we became aware of a related work investigating $\mathrm{Bi}_{2} \mathrm{Se}_{3} / \mathrm{graphene} / \mathrm{Bi}_{2} \mathrm{Se}_{3}$ quantum wells [32]. Although the details are different, we find an agreement for the range of lattice spacing where the two sets of calculations can be compared.

\section{ACKNOWLEDGMENTS}

This work is sponsored by Science Foundation of Ireland (SFI) under the CSET grant underpinning CRANN and under the QDFUN project (Grant No. 07/IN.1/I945). A.N. acknowledges financial support from the Irish Research Council. Computational resources have been provided by the HEA IITAC project managed by TCHPC. 
[1] M. Hasan and C. Kane, Rev. Mod. Phys. 82, 3045 (2010).

[2] H. Manoharan, Nat. Nano. 5, 477 (2010).

[3] H. Zhang, C.-H. Liu, X.-L. Qi, X. Dai, Z. Fang, and S.-C. Zhang, Nat. Phys. 5, 438 (2009).

[4] K. Park, J. J. Heremans, V. W. Scarola, and D. Minic, Phys. Rev. Lett. 105, 186801 (2010).

[5] K. Yang, W. Setyawan, S. Wang, M. B. Nardelli, and S. Curtarolo, Nat. Mater. 11, 614 (2012).

[6] M. König, S. Wiedmann, C. Brüne, A. Roth, H. Buhmann, L. W. Molenkamp, X.-L. Qi, and S.-C. Zhang, Science 318, 766 (2007).

[7] G. Wu, H. Chen, Y. Sun, X. Li, P. Cui, C. Franchini, J. Wang, X.-Q. Chen, and Z. Zhang, Scientific Reports 3, 1233 (2013).

[8] D. Wei and Y. Liu, Adv. Mater. 22, 3225 (2010).

[9] A. Geim and K. Novoselov, Nat. Mater. 6, 183 (2007).

[10] Y.-M. Lin, A. Valdes-Garcia, S.-J. Han, D. B. Farmer, I. Meric, Y. Sun, Y. Wu, C. Dimitrakopoulos, A. Grill, P. Avouris et al., Science 332, 1294 (2011).

[11] J. Li, I. Martin, M. Buttiker, and A. F. Morpurgo, Physica Scripta T146, 014021 (2012).

[12] C. L. Kane and E. J. Mele, Phys. Rev. Lett. 95, 226801 (2005).

[13] C. Weeks, J. Hu, J. Alicea, M. Franz, and R. Wu, Phys. Rev. X 1, 021001 (2011).

[14] W. Dang, H. Peng, H. Li, P. Wang, and Z. Liu, Nano Lett. 10, 2870 (2010).

[15] C.-L. Song, Y.-L. Wang, Y.-P. Jiang, Y. Zhang, C.-Z. Chang, L. Wang, K. he, X. Chen, J.-F. Jia, Y. Wang et al., Appl. Phys. Lett. 97, 143118 (2010).

[16] Y. Jiang, Y. Sun, M. Chen, Y. Wang, Z. Li, C. Song, K. He, L. Wang, X. Chen, Q.-K. Xue et al., Phys. Rev. Lett. 108, 066809 (2012).
[17] G. Kresse and J. Furthmüller, Phys. Rev. B 54, 11169 (1996).

[18] G. Kresse and J. Furthmüller, Comput. Mater. Sci. 6, 15 (1996).

[19] J. P. Perdew, K. Burke, and M. Ernzerhof, Phys. Rev. Lett. 77, 3865 (1996).

[20] P. E. Blöchl, Phys. Rev. B 50, 17953 (1994).

[21] H. Lind and S. Lidin, Solid State Sc. 5, 47 (2003).

[22] J. Furthmüller, J. Hafner, and G. Kresse, Phys. Rev. B 53, 7334 (1996).

[23] W.-K. Tse and A. H. MacDonald, Phys. Rev. B 82, 161104(R) (2010).

[24] M. Vozmediano, M. Katsnelson, and F. Guinea, Phys. Rep. 496, 109 (2010).

[25] Q. Zhang, Z. Zhang, Z. Zhu, U. Schwingenschlögl, and Y. Cui, ACS Nano 6, 2345 (2012).

[26] T. Hirahara, N. Fukui, T. Shirasawa, M. Yamada, M. Aitani, H. Miyazaki, M. Matsunami, S. Kimura, T. Takahashi, S. Hasegawa et al., Phys. Rev. Lett. 109, 227401 (2012).

[27] F. Yang, L. Miao, Z. Wang, M.-Y. Yao, F. Zhu, Y. Song, M.-X. Wang, J. Xu, A. Fedorov, Z. Sun et al., Phys. Rev. Lett. 109, 016801 (2012).

[28] A. R. Rocha, V. M. García-Suárez, S. Bailey, C. Lambert, J. Ferrer, and S. Sanvito, Phys. Rev. B 73, 085414 (2006).

[29] A. W. Robertson, C. Allen, Y. Wu, K. He, J. Olivier, J. Neethling, A. Kirkland, and J. Warner, Nat. Commun. 3, 1144 (2012).

[30] M. Dion, H. Rydberg, E. Schroder, D. C. Langreth, and B. I. Lundqvist, Phys. Rev. Lett. 92, 246401 (2004).

[31] G. Cunningham, M. Lotya, C. Cucinotta, S. Sanvito, S. Bergin, R. Menzel, M. Shaffer, and J. N. Coleman, ACS Nano 6, 3468 (2012).

[32] L. Kou, B. Yan, F. Hu, S.-C. Wu, T. O. Wehling, C. Felser, C. Chen, and T. Frauenheim, Nano Lett. 13, 6251 (2013). 